\title{
DIE GESCHICHTE DER BILDUNG UND WISSENSCHAFTLICHEN FORSCHUNG IN BRASILIEN
}

\section{ORIGINAL-ARTIKEL}

FREITAS, Roberto Araujo de Moraes ${ }^{1}$

MARTINS, Priscila Bernardo ${ }^{2}$

FREITAS, Roberto Araujo de Moraes. MARTINS, Priscila Bernardo. Die Geschichte der Bildung und wissenschaftlichen Forschung in Brasilien. Revista Científica Multidisciplinar Núcleo do Conhecimento. 04-Jahr, Ed. 12, Vol. 08, S. 127-138. Dezember 2019. ISSN: 2448-0959,

Zugangslink: https://www.nucleodoconhecimento.com.br/bildung-de/geschichte-derbildung

\section{ZUSAMMENFASSUNG}

Im aktuellen Artikel werden die historischen Veränderungen der Lehrtätigkeit in Brasilien sowie ihre Teilnahme an der akademisch-wissenschaftlichen Forschung thematisiert, wobei von den Einflüssen, die sich aus den ererbten Religionen aus Europa ergeben, bis hin zu den Kennzeichen der Ressourcen Technologien in den Händen der inländischen Nutzer. Dieser Moment ist durch den Übergang zwischen dem 20. und 21. Jahrhundert gekennzeichnet und stellt somit eine grassierende Revolution im technologischen Sektor dar, wobei das Internet das wichtigste Instrument der Kommunikation und Forschung ist, was zu einem langen

1 Postgraduierter in der Lehre in der Hochschulbildung, graduierte in Computer Engineering.

2 Doktorat in Naturwissenschaften. Master-Abschluss in Naturwissenschaften Lehre. Spezialisierung im Fernunterricht: Vorbereitung von Materialien, Nachhilfe und virtuelle Umgebungen. Spezialisierung in Wirtschaftspädagogik. Abschluss in Mathematik. Abschluss im Personalmanagement. Abschluss in Pädagogik. 
Anpassungsprozess geführt hat. Es ist notwendig, die Schwierigkeit zu erwähnen, die es im Prozess der Isolierung des Wissens über seine lokale Kultur, Meinungen und Erfahrungen seiner eigenen und des akademischen Gepäcks, mit dem ein Lehrer an die Studentenschaft zu übertragen, weil er verstehen muss, dass die Brasilien verzeichnet Einflüsse verschiedener Völker auf der ganzen Welt, was uns zu einer solchen Gemischten Kultur gemacht hat. Inmitten so vieler historischer Veränderungen muss die Lehrtätigkeit ihre Methoden ständig recyceln lassen. Aus diesem Grund wird diese Arbeit anstrengender, da der Lehrer unter den Auswirkungen des Netzwerks auf die Welt leidet, da sie sich in der Produktivität und Zuverlässigkeit der Ergebnisse literarischer und wissenschaftlicher Arbeiten widerspiegelt. Dies wird das Verhältnis zwischen Wissen und Information für immer gefährden.

Schlagworte: Technologie, Forschung, Bildung, Zuverlässigkeit.

\section{EINFÜHRUNG}

Die Ausübung des Unterrichts hat immer gesellschaftliche Veränderungen durchgemacht, insbesondere nach den ideologischen Interessen jeder Region, entweder aufgrund religiöser oder politischer Natur. Aus der Geschichte Brasiliens, die aus dem Jahr 1500 stammt, ist der Einfluss des Christentums in den ersten Schulen des Landes, orchestriert von der europäischen Institution companhia de Jesus, relevant. Im Laufe der Jahre gab es Anreizzeiten sowie Vernachlässigung des Bildungsniveaus der Bevölkerung. Diese Lücke ist eine Realität, die bis heute nicht überwunden ist, vor allem, weil es möglich ist, sehr geringe Investitionen in den akademischen Sektor sowie in die wissenschaftliche Forschung zu tätigen, auch wenn es Gesetze gibt, die den Sektor in gewisser Weise unterstützen.

Gesetz, das in Brasilien Empire geboren wurde, genauer gesagt in der Regierung von D. Pedro I. Das vorgenannte wurde als Gesetz der Richtlinien und Grundlagen bezeichnet, ein Gesetz, das sich bis heute in allen Regierungen ändert und seine neueste Version aus dem Jahr 1996 ist. Der pädagogische Traditionalismus, der von Bibliotheken, Büchern, Artikeln, Leitartikeln usw. gegeben wird, beginnt sich am Ende des 20. Jahrhunderts mit der Agglomeration von Informationen im Internet zu 
verändern. Dies spiegelt sich in der Schwierigkeit wider, die Lehrer und Forscher bis heute haben, sich an neue Arbeitstrends anzupassen, da sie in dem Maße, wie sie den Zugang zu Informationen erleichtern, die Zuverlässigkeit der Inhalte drastisch verringern.

\section{DIE GESCHICHTE DER BILDUNG UND WISSENSCHAFTLICHEN FORSCHUNG IN BRASILIEN}

\subsection{ENTDECKUNG UND KOLONISIERUNG}

Die ersten bedeutenden Indikatoren der brasilianischen Bildung, im Sinne der akademischen Schule, werden durch die Geschichte des Landes, von seiner Entdeckung durch die Regierung von Portugal, offiziell im Jahr 1500, parallel. Seit jeher hat der Zugang zu Informationen und die Art und Weise, wie Menschen in Brasilien geschaffen und ausgebildet werden, Auswirkungen auf das Verhalten der Gesellschaft, weil sie nach dem Wunsch der herrschenden Klasse manipuliert wird. Laut Ribeiro (1993) war es für Siedler nicht interessant, den Einheimischen Zugang zu Informationen zu gewähren, da es sich um eine Strategie handelte, die in der Lage war, den Schock divergierender Kulturen und Interessen zwischen dem Hof und der indigenen Bevölkerung zu provozieren.

Der Vorschlag war a priori, Einzelpersonen der lokalen Masse blind und unterwürfig mit dem Werk zusammenarbeiten zu lassen, und dieses Ziel wurde von den Europäern auf der Grundlage der Verzerrung der kulturellen und religiösen Identität der einheimischen brasilianischen Bevölkerung entworfen. Aus diesem Grund hatten sie kaum Zugang zu Alphabetisierung, und so wurde ihre Kultur gewaltsam manipuliert. Während dieses Prozesses wurden mehrere indigene Nationen dezimiert, weil sie sich dem Jesuitenprozess nicht unterwerfen konnten. Von Europa aus hatten die Siedler die Unterstützung der Jesuiten, um das Christentum in Brasilien zu verbreiten, wie sie es in mehreren Ländern über ihre Elterninstitution, die Gesellschaft Jesu, taten. Die Organisation hat mehrere grundlegende Bildungseinheiten in Brasilien errichtet. 
Einundzwanzig Jahre nach der Ankunft der Jesuiten könnte der Orden der Gesellschaft Jesu bereits fünf Grundschulen an verschiedenen Orten im ganzen Land haben, vor allem in Küstenregionen: Porto Seguro, Ilhéus, Espérito Santo, Sao Vicente und Sao Paulo von Piratininga. Mit innen drei Schulen: Salvador, Rio de Janeiro und Olinda (ALVES, 2009). Hier tritt der Unterschied zwischen Schule und Schule je nach Bildungsniveau auf. Lesen Sie daher eine Schule für Grundschuleinrichtungen (Erste Klasse oder Grundschule I und II, gemäß der Nomenklatur ldb/96) und eine Sekundarschule (Zweiter Grad oder Gymnasium, gemäß der von LDB/96 eingerichteten Nomenklatur).

Die Jesuiten waren etwa zweihundert Jahre lang für die Bildung in Brasilien verantwortlich. Erstens, die Katechisierung der Indianer, die sie Wilde nannten. Später gründeten sie Schulen und Missionen. Dieser Prozess der Verbreitung westlicher Lehren folgte bis zur Vertreibung der Jesuiten 1759. Damit trat der Bildungsprozess in eine Phase der Stagnation ein. Laut Rosa (2014) sank der Orden der Gesellschaft Jesu nach etwa 200 Jahren der Ankunft der Portugiesen in Brasilien. Zu dieser Zeit war Der Marquis von Pombal, Sebastian José de Carvalho e Melo, Staatsoberhaupt und internationaler Diplomat Portugals.

Laut NISKIER (1969) wurde aufgrund der Unabhängigkeitserklärung sogar versucht, das Bildungssystem durch Artikel 250 zu erweitern, der die Schaffung von Grundschulen in jedem Dorf, Turnhallen in jedem Bezirk und Universitäten an bestimmten Orten, aber dies war nur in der Theorie, weil der Kaiser löste den Konstituenten und im folgenden Jahr gab er die erste Verfassung des Reiches und in Artikel 179 sagte: "Grundschulbildung ist frei für alle Bürger." 1827 erließ sie das Allgemeine Gesetz, das bis 1946 das erste und einzige für die öffentliche Grundschulbildung sein wird. D. Pedro II. regiert zwar seit 49 Jahren als sehr kultisch, aber seine Projekte im Bildungssektor waren zaghaft und ohne Relevanz (ALVES, 2009, S. 10).

Als Mitglied der portugiesischen Elite störte er ihn sehr über den Aufstieg des religiösen sozialen Bereichs des Ordens, da er die politischen Befugnisse der Regierung 
ausdemaierte. Als Ergebnis, am 22. Januar 1759, wurde der Satz veröffentlicht, der die Befreiung und Denaturalisierung von Jesuiten aus dem Gebiet Portugiesisch (einschließlich Brasilien) bestimmt. Die Situation änderte sich erst mit der Ankunft der königlichen Familie. Während der Zeit der Monarchie spürt die Erziehung die Reflexionen der Vertreibung der Jesuiten. Don Pedro I. regiert seit Jahren, hat aber keine Neuigkeiten.

\subsection{SOZIALE INVESTITIONEN IM BILDUNGSSEKTOR UND DER EINFLUSS VON PROFESSOR PAULO FREIRE}

Aufgrund der langen Zeit, die das Allgemeine Gesetz dauerte, erlitt der Bildungssektor in Brasilien erst ab 1946, nach der Absetzung von Getélio Vargas, während der Zeit, die als Zweite Republik bekannt wurde: als die Diktatur der Vargas ging zu Ende und das brasilianische republikanische Regime wurde wieder aufgenommen. 1948 begannen nach den Vorgaben der fünften Verfassung die Arbeiten am Gesetz der Richtlinien und Basen, das 1961 verkündet wurde. Zeitgleich mit der Verkündung der LDB entstand die Volksbildungsmethode von Professor Paulo Freire. Die Populärkulturbewegung MCP, geleitet von Paulo Freire selbst, hatte Bauern als erste Schüler, die diese Erfahrung durchgemacht haben, indem sie von innen nach außen durch ihre eigene Arbeit gebildet wurden. Die erzielten Ergebnisse - 300 gebildete Arbeitnehmer in 45 Tagen - haben die öffentliche Meinung tief beeindruckt.

Es wurde beschlossen, die Methode im gesamten Staatsgebiet anzuwenden, wurde aber dieses Mal von der Bundesregierung unterstützt. Seit der Einführung der LDB sind die Investitionen in die Bildung bemerkenswert gestiegen. Die Popularisierung der Lehrmethoden von Professor Paulo Freire inspirierte mehrere Menschen und Institutionen und schuf so neue Projekte und soziale Gruppen, um die Verbreitung von Ausbildung und Wissen zu unterstützen. Natal/RN war die erste brasilianische Stadt, die diese Didaktik mit der Alphabetisierungskampagne "Standing On The Ground Also Learns To Read" anwendete, die sich an an an Gebildete richtete. Alves (2009, S. 67) sagt, dass es zu dieser Zeit auch "die Volkszentren der Kultur, verbunden mit der 
National Union of Students; die Grassroots Education Movement (verbunden mit der Nationalen Bischofskonferenz Brasiliens) und die Regierung der Union."

1962 wurde der Eidgenössische Bildungsrat in Übereinstimmung mit Artikel 9 des Richtlinien- und Bemessungsgesetzes gegründet. Dies tritt an die Stelle des National Board of Education. Im selben Jahr werden die staatlichen Bildungsräte, der Nationale Bildungsplan und das Nationale Alphabetisierungsprogramm geschaffen, die beiden letztgenannten vom Ministerium für Bildung und Kultur, auf Vorschlag der Paulo Freire Methode. Mit der Schaffung dieses Systems eröffnet Freire eine neue Stufe der Erwachsenenbildung in Brasilien. Dieses Bildungsprojekt, das vom Cultural Extension Service der Universität Recife unter seiner Leitung, dem Paulo Freire Education System, durchgeführt wurde, hatte als Hauptziel des Analphabetismus für Erwachsene. Nach kurzer Umsetzung wurden diese Projekte jedoch durch den Militärputsch von 1964 unterbrochen. So, in den Worten von Oliveira (2001, S.56):

Bis zum Staatsstreich vom 31. März 1964 war die Beziehung zwischen progressiven Regierungen und Volksbewegungen, die sich auf Volksbildung konzentrierten, kollaborativ. Das heißt, um Anstrengungen zu unternehmen, um die Vorschläge des brasilianischen Populismus und Entwicklungssatik umzusetzen. Einige der ausdrucksstärksten Bewegungen der Bildung und der Populärkultur in Brasilien werden dort geboren. Dies sind Ausdruck dieser Bewegung: Die Volkskulturbewegung (MCP), die Grassroots Education Movement (MEB), das Popular Center of Culture (CPC) und die Kampagne "Standing on the Ground" lernen auch lesen." Von da an waren Bildung und Kultur nicht mehr auf die einfache Wählerbildung zurückzuführen. Instrumente der Transformation der sozialen Struktur werden als Räume für die Bildung bewusster, kritischer und teilnehmerischer Wesen betrachtet.

Mit dem Militärputsch 1964 geht es für die sozialen Bewegungen stark unter Druck, was auch auf die Gruppen und Institutionen wirkt, die an Volksbildungs- und Kulturprojekten mitgearbeitet haben. Die MEB blieb jedoch vom National Literacy 
Program in Betrieb. Zwischen 1970 und 1971, als Brasilien bei der Gründung des institutionellen Aktes Nr. 05 in der Regierung Costa Silva in die repressivste Periode der Militärdiktatur eintrat, eine Periode, die als "Putsch innerhalb des Putsches" bezeichnet wurde. Während dieser Zeit entschied sich die Regierung für die Installation einer noch größeren Struktur, der brasilianischen Alphabetisierungsbewegung - MOBRAL (Gesetz Nr. 5. 379 de 15/12/67).

Zu seinen Zielen gehörte die Verbreitung der offiziellen Ideologie der Entwicklung, um das gegenwärtige Modell der Herrschaft und Modernisierung zu stärken. Nach den Worten von Bittar und Bittar (2012, S. 163) waren die Regierungen nach der Diktatur jedoch auch nicht in der Lage, das Problem zu lösen, und darüber hinaus "weil sie die Universalisierung der Grundschule nicht eingehalten haben, eine Aufgabe, die von den meisten Ländern erfüllt wurde. Vom 19. bis zum 20. Jahrhundert trat Brasilien mit diesem schändlichen Erbe in das 21. Jahrhundert ein."

\subsection{FORSCHUNGSANREIZEINRICHTUNGEN}

Auch in den frühen 1960er Jahren sah ein Teil der Regierung einen Vorteil bei Investitionen in die technologische und wissenschaftliche Entwicklung. Während dieser Zeit wurde fapesp - Sao Paulo State Research Support Foundation gegründet, und mit inm mehrere andere verwandte Institutionen Brasilien innerhalb. Zu den Zielen gehörte die Schaffung des strictu sensu Graduiertenmodells. Bemerkenswert ist auch die SBPC - Brasilianische Gesellschaft für den Fortschritt der Wissenschaft, gegründet 1934, in der Stadt Sao Paulo / SP, die aber begann, mehr Sichtbarkeit und Autonomie im Übergang zwischen den 1960er und 1970er Jahren zu haben. 1988 verkündete Brasilien seine neue Verfassung, die so genannte Bürgerverfassung, deren Artikel 208 festlegt, dass es die Pflicht des Staates ist, die obligatorische und kostenlose Grundschule zu garantieren.

[...] es war ein wichtiger historischer Meilenstein in der brasilianischen Bildung, da dieses Gesetz die Schulbildung umstrukturierte und die verschiedenen Bildungsniveaus und -modalitäten neu ausrichtete. [...]einen Prozess der Umsetzung von Reformen, Politiken und 
Bildungsmaßnahmen, anstat[...]t den privaten Expansionsprozess zu bremsen und die Richtungen der Hochschulbildung zurückzurichten, trug zum genauen Gegenteil bei: Es expandierte und führte eine ein diversifiziertes und differenziertes System, vor allem durch Zugangsmechanismen, akademische Organisation und angebotene Kurse. In diesem Zusammenhang schuf er die sogenannten sequentiellen Kurse und Universitätszentren; die Zahl der Universitäten, die sich auf den Wissensbereich spezialisiert haben; implementierte Zentren für technologische Bildung; das Vestibuläre durch selektive Prozesse ersetzt; die Mindestlehrpläne zu beenden und die Lehrpläne zu lockern; erstellte Technologiekurse und Hochschulen, unter anderem Änderungen (BITTAR; BITTAR, 2012, S. 165).

Es lohnt sich auch, einige Jahre später die Politik zu wiederholen, die in beiden Mandaten der Regierung von Präsident Fernando Henrique Cardoso angenommen wurde, vor allem die Zunahme der Einschreibungen in brasilianische öffentliche Schulen. Es war eine Politik, die darauf abzielte, mehr Schüler von der Grundschule bis zum Gymnasium anzuziehen. Auf der anderen Seite hat der Staat aufgehört, der Hochschulbildung Priorität einzuräumen, was automatisch zu einem Mangel an Ressourcen und auch Anreizen führte. Diese negative Situation führte dann zur Vorzeitigseinlage von Lehrern und zu höheren Inzidenzen bei ihren Transfers in gleichwertige private Sektoren. In diesem Zusammenhang begann die Regierung mit der Ausarbeitung einer neuen Fassung für das Guidelines and Bases Act von 1996.

\subsection{ZWISCHEN ACADEMIC ENCYCLOPEDIAS UND VAST INTERNET CONTENT FÜR WISSENSCHAFTLICHE FORSCHUNG}

Forschung ist seit den 1960er Jahren in Schulen üblich, mit dem Vorschlag, die Schüler in Akteure ihres Bildungsprozesses zu verwandeln. Die Forschung allein garantiert jedoch nicht die kritische Entwicklung der Schüler und hängt von der pädagogischen Praxis ab, die vom Lehrer geleitet wird. Garcia (2017) erklärt, dass der Forschungsprozess exponentiell nach der Domestizierung von Mikrocomputern und 
dem Internet erleichtert wurde. Aber dies bedeutete einige Störungen für die Wissenschaft und das schulische akademische Umfeld: die Wegwerfbarkeit von Informationen. Viele Informationen sind nicht immer gleichbedeutend mit viel Wissen. Da Informationen mit wenigen Klicks erreicht werden können, ist das Interesse am Lernen zurückgegangen. Bis dahin erforderte die Ausübung einer Schularbeit viel mehr Aufwand, da der Schüler ein paar Stunden in einer Bibliothek verbringen sollte, um in zahlreichen Büchern nach den gewünschten Informationen zu suchen.

Um den Prozess zu erleichtern, gab es Enzyklopädien, die verschiedene Themen präsentierten, die später durch Ausgaben in Kiosken verkauft wurden. Derzeit ist das Internet die größte Datenquelle für die Schulforschung. Dies begann mit der Kommerzialisierung von Mikrocomputern in Heimumgebungen, die nicht lange vor dem Zugriff auf das Netzwerk waren (FERRARESI et al., 2009). Bis dahin behielten Universitäten und Schuleinrichtungen die Kultur der Förderung der Forschung zu dem, was in Büchern, Enzyklopädien, wissenschaftlichen Artikeln usw. verfügbar war. Bis heute haben Lehrer große Schwierigkeiten, ihre Arbeit an die aktuellen technologischen Ressourcen anzupassen, da das Internet ein Umfeld ist, das sich ständig ausdehnt und transformiert. Da die Zahl der Cyber-Nutzer exponentiell auf dem ganzen Planeten wächst und wenn man bedenkt, dass sie alle die Leichtigkeit der Produktion und Extraktion von Inhalten aus dem Netzwerk genießen, erfordert die Nutzung des Internets als Forschungsquelle für akademische Arbeit eine Menge Pflege.

Es ist erwähnenswert, dass die selbst zunächst für akademische Zwecke geschaffen wurde. Das akademisch-wissenschaftliche Umfeld beschäftigt sich mit dem Grad der Glaubwürdigkeit angesichts der aus dem Netz gewonnenen Informationen. Aufgrund der vorgeschlagenen Unmittelbarkeit, die mit der neuen Kultur der elektronischen Sofortkommunikation verbunden ist, ist der Anteil der Studenten und Forscher, die immer noch Bibliotheksbücher, gedruckte Artikel und dergleichen für das Schreiben ihrer Thesen nutzen, minimal. In jedem Fall folgten die Universitäten in der Regel mit der Entschlossenheit, ihre Bibliotheken auf dem neuesten Stand zu halten. Das Internet änderte nichts an der Glaubwürdigkeit der Bücher, so dass diese weiterhin die 
angesehensten bei der Suche nach Informationen waren. Ferraresi et al (2009, S. 4) behauptet:

Studien- und Suchaktivitäten über Online-Kommunikation werden von Suchseiten aus durchgeführt, wobei die Suchseite Google (...) vorherrschen. Und unter den Seiten, die die Befragten kennen (...) universiabrasil, ibge und schoolar.google werden mehr zitiert.

In diesem neuen Moment fassen Ramos und Copolla (2009) zusammen, dass das akademische Umfeld dem technologischen Zeitalter gerecht werden muss, und passen ihre Innenräume an diese Realität an. Das Internet kann für verschiedene Zwecke genutzt werden, und die Auszubildenden müssen geschult werden, um von inm aufgrund seiner Fragilität präzise und zuverlässige Informationen zu erhalten, da jeder Benutzer Inhalte produzieren kann. Es sollte eine Analysearbeit durchgeführt werden, um das Internet als ein pädagogisches Werkzeug zu überprüfen, entsprechend den Zielen des Benutzers zu einem bestimmten Zeitpunkt. Sie erklären, dass das Internet in reichweite des Heimnutzers erlaubt, KlassenzimmerBarrieren zu brechen und die Entfernungen zwischen dem Lehrer und dem Schüler zu verkürzen, wenn er nicht auf einen festen Zeitplan angewiesen sein kann oder will, und damit Bildung profitiert von der Kollektivität.

(...) das Internet (...) ist zu einer potenziell unendlichen Informationsquelle geworden und hat dem Lehrer verschiedene Arten von Vorteilen und neue Probleme gebracht, denn während es viel anspruchsvollere und noch interessantere pädagogische Interventionen ermöglicht dass traditionelle Klassen (...), den Lehrer dazu bringen, Veränderungen in den Machtverhältnissen über wissen zu begegnen: wo der Lehrer aufhört, die Kontrolle über den Referenzinhalt zu haben, und beginnt, unvorhersehbare Informationen zu verwalten (...). Aus diesem Grund sollte sie sich für den Einsatz dieser Instrumente in der Bildung nicht auf die Lehrerausbildung als eine weitere technologische Innovation beschränken (RAMOS; COPOLLA, 2009, S. 7). 
Es fördert die Fähigkeit zum Selbstlernen, sollte aber nicht als Allheilmittel angenommen werden, wie es bis dahin mit dem Lehrbuch geschehen war. Das bedeutet, dass technologische Ressourcen, so sehr sie in die akademische Realität gebracht werden, nicht als das einzig mögliche Arbeitsmittel für die Durchführung von Aktivitäten verstanden werden können, sondern zu Dynamik und Kreativität sowie stellt eine Verbindung zwischen den theoretischen Konzepten und dem Privatleben jedes einzelnen Schülers her. Das Internet ist eine unendliche Informationsquelle, und dies führt dazu, dass der Lehrer das Bild des Informationsinhabers verliert. Es ist daher notwendig, beim Umgang mit diesen Informationen etwas Vorsicht walten zu lassen.

\subsection{DIE ROLLE DES LEHRERS IN DER METHODOLOGISCHEN TRANSFORMATION DER AKADEMISCHEN UMWELT}

Es ist unbestritten, dass der Lehrerberuf flexibel wird, da die Struktur der gesamten Gesellschaft von ihren kulturellen Traditionen und Ressourcen abhängt, die für die Arbeit zur Verfügung stehen. Der Mensch treibt in der Moderne seine Arbeit an, um alle Bereiche der Gesellschaft miteinander zu verbinden. Aus diesem Grund sollte der gute Lehrer seine Arbeit auf der Suche nach ständiger Erneuerung dessen, was er zu bieten hat, mit dem Ziel, die Leistung und Qualität des Dienstes zu erhöhen, zugegeben, dass die Einbeziehung neuer Werkzeuge in die Realität des Klassenzimmers Aspekte bringen kann Positive. In diesem Zusammenhang zeigen Ramos und Copolla (2000, S. 9) in ihrer Studie darauf an, dass:

Der Lehrer nimmt eine neue Haltung ein. Obwohl er immer wieder immer noch die Rolle eines Spezialisten spielt, der über Kenntnisse und/oder Erfahrungen verfügt, um zu kommunizieren, wird er häufiger die Rolle des Beraters für die Aktivitäten des Studenten, Beraters, Lernbegleiters von jemandem spielen, der zusammenarbeiten kann, um das Lernen der Schüler zu fördern, die Rolle derjenigen spielen, die als Team arbeiten, zusammen mit dem Studenten, der die gleichen Ziele sucht: ein Wort, wird die Rolle der pädagogischen Vermittlung entwickeln. 
Die Computerisierung des Unterrichts allein reicht nicht aus, um Wissen zu produzieren. Der Lehrer ist in erster Stelle ein Überträger des Wissens. Er muss sein gesamtes akademisches Gepäck nutzen, um Interesse an dem Studenten für die Forschung zu wecken. Es ist bekannt, dass das Internet, wenn es richtig genutzt wird, eine Quelle des Wissens ist. Wenn sie jedoch wahllos und ohne Anleitung verwendet wird, kann dies einen Bärendienst für Informationen darstellen. Akademische Arbeiten, bei denen der Student nur das weltweite Computernetzwerk als Forschungsquelle für das Schreiben seiner Arbeit nutzt, können zu harmloser Arbeit führen. An dieser Stelle merken wir die Bedeutung der Anwesenheit des Lehrers, denn er sollte beim Schüler die Neugier wecken, über eine einfache Lektüre auf Wikipedia hinauszugehen.

Das Fakultätsteam des akademischen Umfelds selbst lehnt die Verwendung bestimmter Websites für die Annahme der Essays seiner Studenten in ihren wissenschaftlichen Artikeln ab, da Informationen, wenn sie im Internet veröffentlicht werden, sehr leicht manipuliert werden können, da die Leichtigkeit, mit der ein verbundener Benutzer auch Inhalte produziert. Auf der anderen Seite bringt dieses neue Szenario Momente des Ungleichgewichts in seiner Arbeit mit sich, da der Schüler nicht mehr nur an den ihm präsentierten Inhalten festhält, sondern auch mit anderen Fragen als der Handlung der Klasse erscheinen kann, basierend auf dem Inhalt. im Internet verfügbar. Für Ramos und Copolla (2000) ist der Lehrer ein Agent, der ständig die Aktualisierung und Wiederverwertung seines Wissensgepäcks suchen muss.

(...) Die Ausbildung sollte immer den pädagogischen Kontext berücksichtigen, in den der Lehrer eingefügt wird, so dass er sie in seine Bekanntwerden und Diskussion über den Einsatz dieser Technologien einbezieht, notwendig ist. Jedem Lehrer in seiner Ausbildungs- und Handlungswirklichkeit die möglichkeit, diese Werkzeuge bewusst zu integrieren und nicht als einfache Apparate, um ihre Klassen zu animieren oder zu veranschaulichen (RAMOS; COPOLLA, 2000, S. 12).

Es ist bekannt, dass Technologien Prozesse beinhalten, die dynamisch und unterbrechungsfrei verbessert werden, was dazu führt, dass ihre Benutzer und insbesondere ihre Entwickler sich engagieren, um mit den aufgetretenen 
Transformationen Schritt zu halten. Im akademischen Umfeld ist es im Allgemeinen nicht wesentlich, dass sich die Lehrer um die Architektur der Entwicklung technologischer Ressourcen kümmern, aber es ist interessant, dass sie weiß, wie sie diese Ressourcen an die Realität des Klassenzimmers veranlassen kann, damit sich die Schüler in Informationsgesellschaft, auch wenn sie sich im akademischen Schulumfeld befinden. Immerseing die Lektion in einer virtuellen Realität bringt den Schülern einen praktischen Blick auf die angesprochenen Themen und macht sie kritischer, wenn sie richtig präsentiert. Wenn der Lehrer das Ziel erreicht, Kreativität, Neugier und Interaktion in den Schülern zu entwickeln, wird er die Inhalte besser nutzen.

\section{ABSCHLIEßENDE ÜBERLEGUNGEN}

Als Ausgangspunkt war die Geschichte der Bildung in Brasilien wichtig, um die Einflüsse der Bevölkerung im Interesse der akademischen Forschung abzugrenzen. Obwohl die brasilianische Bildung gesetzlich geregelt ist, hat sie seit ewigen Zeiten nur wenige Investitionen in Forschung und Bildung getätigt, und dies ist auf politische Interessen zurückzuführen. Professor Paulo Freire versuchte sogar, seinen Beitrag zu verlassen und das tragische Bild des Analphabetismus in Brasilien umzukehren. Dasselbe wurde international erwähnt und von den großen Nationen und Universitäten beklatscht,

Hier wurde das Projekt zerstört und viel kritisiert. Im 21. Jahrhundert sieht der Lehrer Schwierigkeiten, sich an die neuen Gesellschaftlichen Tendenzen in Bezug auf das Internet anzupassen, Bibliotheken und Leitartikel zu ersetzen und die Glaubwürdigkeit der Urheberschaft des Werkes zu verringern, da der Akt des Unterrichts nicht direkt mit dem technologischen Universum, aber das ist es, was alle Sektoren der Gesellschaft und am Arbeitsplatz globalisiert. 


\section{REFERENZEN}

ALVES, W. L. U. A história da educação no Brasil: da Descoberta à Lei de Diretrizes e Bases de 1996. 2009. 76 f. Monografia (Especialização em Metodologia do Ensino Superior) - Centro Universitário Católico Salesiano Auxilium. Lins, 2009.

BITTAR, m; BITTAR, M. História da educação no Brasil: a escola pública no processo de democratização da sociedade. Acta Scientiarum Education, v. 34, n. 2, p. 157168, 2012.

FERRARESI, A. C. et al. Uso da internet como fonte de pesquisa entre universitários: um estudo de caso. In: XIII Encontro Latino Americano de Iniciação Científica e IX Encontro Latino Americano de Pós-Graduação - Universidade do Vale do Paraíba, 2009

GARCIA, R. Antes do Google e da Wikipédia - as enciclopédias do passado. 2017. Disponível em: https://vejasp.abril.com.br/blog/memoria/antes-do-google-e-dawikipedia/. Acesso em: 17 dez. 2018.

OLIVEIRA, E. S. Diferentes sujeitos e novas abordagens da educação popular urbana. 2001. 152 f. Dissertação (Mestrado em Educação) - Universidade Federal Fluminense. Niterói, 2001.

RAMOS, M; COPOLLa, N. C. O uso do computador e da internet como ferramentas pedagógicas. PDE. 2009. Disponível em: http://www.diaadiaeducacao.pr.gov.br/portals/pde/arquivos/2551-8.pdf. Acesso em: 17 jan. 2019.

RIBEIRO, P. R. M. História da educação escolar no brasil: notas para uma reflexão. Paiadéia, n. 4, p. 15-30, 1993.

ROSA, T. F. lluminismo e a expulsão dos jesuítas do Império Português; as reformas pombalinas e o plano dos estudos menores. Revistas de História Regional, v. 19, n. 2, p. 361-383, 2014. 
Eingereicht: August 2019.

Genehmigt: Dezember 2019. 